\title{
Rendering 3D graphics as an aid to stylized line drawings in perspective
}

\begin{abstract}
The aim of the research was to study the issue of drawing 2D objects and environments in perspective and attempted to ease the process of drawing them with the aid of three-dimensional computer graphics. The goal of the research was to develop the method, which would exclude the need to trace three-dimensional models, which many digital artists use as a guide when making drawings. The need to trace has been eliminated by finding a procedure to render three-dimensional models to appear drawn - to appear drawn by an artist who has a stylized line style. After researching various techniques of rendering, Sketchup was used to make and apply a Sketchup style which emulated a line style. After that, various tests were performed using computer measurements and questionnaires to determine if the observers could distinguish between three-dimensional renders and two-dimensional drawings. The results have shown that very few participants notice three-dimensional graphics rendered with Sketchup. Even among the few observers who did notice the presence of three-dimensional models, none detected even half. The results confirmed the adequateness of the methodology, which enables a more correct creation of element in perspective and convinces the observers that the entire image is stylistically uniform hand drawn image.
\end{abstract}

\author{
Mark Arandjus, \\ Helena Gabrijelčič Tomc \\ University of Ljubljana, \\ Faculty of Natural Sciences and \\ Engineering, Ljubljana, Slovenia \\ Corresponding author: \\ Helena Gabrijelčič Tomc \\ e-mail: \\ helena.gabrijelcic@ntf.uni-lj.si
}

First recieved: 24.12.2015. Accepted: 26.10.2016.

\section{KEY WORDS}

3D art, style, perspective, method, non-photorealistic rendering, Sketchup

\section{Introduction}

Creating perspective in art is a difficult task. It is quite technical and does not allow any artistic liberty due to its exactly prescribed rules. In addition, the human eye is extremely used to observing perspective, making it quite sensitive to irregularities in drawings. These factors make perspective drawings demanding in terms of precision, even at the basic level.

To ease the creation of perspective drawings artists (particularly digital artists) use 3D models. 3D art software has become very accessible and easy to use. Artists can, with little knowledge of 3D graphic theory and not much effort, make more or less complex 3D models of objects and environments. The models are then exported as a $2 \mathrm{D}$ graphic and are used as guides when drawing perspective through the process of tracing. Thus artists have unburdened themselves with the technical aspects and difficulties of drawing perspective, but have consequently created a new problem: tracing. Renders of 3D models are very geometrically correct and most artists do not draw clean straight lines. Moreover, many artists embrace their drawing imperfections, as they are often unique to them and contribute to the recognition of their style. In using 3D graphics artists no longer have to deal with the strains of drawing perspective but the use of 3D means they must trace renders, if they want the models to stylistically uniform to the hand drawn parts of the image. Rendering 3D computer models to imitate $2 \mathrm{D}$ art has been in use in the entertainment industry, such as animated films and computer games 
for several years. A couple of notable examples include the surface of the magic carpet in Disney's Aladdin (Aladdin, 1992, The Making of 'Aladdin': a Whole New World, 1992), the robot from The Iron Giant (The Iron Giant, 1999), the deep canvas process used to render the trees in Disney's Tarzan (Tarzan, 1999, Walt Disney Studios, 2000), cars in the TV series Family Guy (Family Guy, 2010), and the general aesthetic in the video game Ni no Kuni (Ni no Kuni, 2010). Rendering of 3D models, as seen in The Iron Giant, fall into the category of so-called non-photorealistic rendering, NPR (Robertson, 1997).

The problem however, with non-photorealistic rendering like this, is that it either pertains to painting techniques (such as the deep canvas process in Tarzan) or it is too geometrically correct to be stylistically seamless with stylized hand drawn art. There have however been advances in the use of 3D graphics to emulate a hand drawn appearance. One such example is the Academy Award winning short Paperman by Disney (Paperman, 2012), which used in-house software called Meander. Meander works by analyzing hand-drawn key frames drawn on top of 3D models and tweening the drawn lines to adjust to the animated models beneath, according to the motion of the 3D graphics (Wilson, 2013). This is then more than just integrating 3D objects into $2 \mathrm{D}$ images, but using $3 \mathrm{D}$ and $2 \mathrm{D}$ graphics on the same element in the shot. Despite great results, Meander was designed to aid the process animation, not actual drawing. As a result, drawing is still a necessity, something we're trying to bypass in our research. Not to mention that it is exclusive software and requires specialization, making it unavailable and unfit for the average artist.

Non-photorealistic rendering is in continuous development by the developers and researchers. Its implementation on 3D geometry include stylization or abstraction and various techniques are used (Bousseau et al., 2007; Kalinins et al., 2009, Kang, Lee \& Chui, 2009; Kyprianidis, Kang \& Döllner, 2009). The NPR studies are in focus especially in 2D and 3D graphic. Author Way with his colleagues (Way et al., 2014) presented a new method in a framework of a pencil style drawing by observing artist's actual drawing process. An example of a rendering 3D models to appear stylized and hand drawn include can include distorting the edges of models (Figure 1) or using predictive algorithms to render the surface of a 3D model based on its lighting and material properties (Kim et al., 2008) (Figure 2). While these methods can be very effective, it is difficult to precisely define how these edges should be deformed. As a result, artists must adjust their style to the software to achieve stylistic unity. In general, the most frequent challenges that the developers encounter in developing algorithms for non-photorealistic visualizations are representation of contours, details and models' silhouettes and non-photorealistic gradients and highlights.

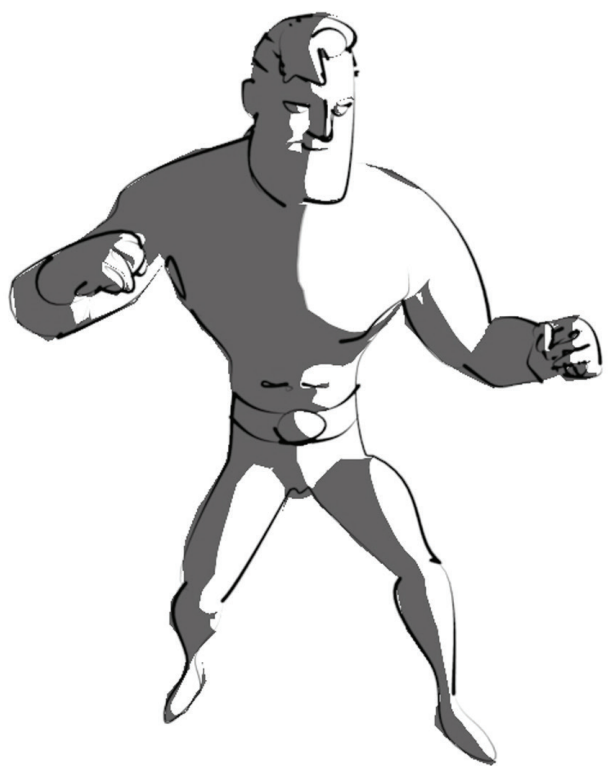

» Figure 1: Non-photorealistic render made using Blender

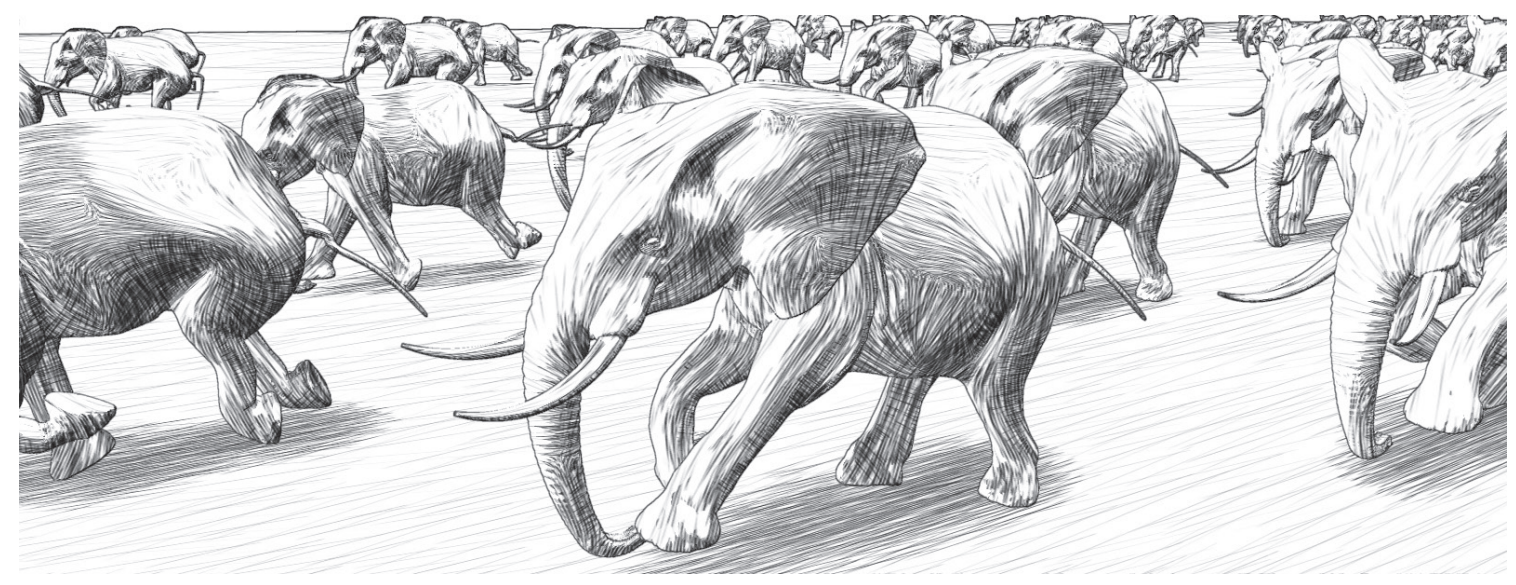

» Figure 2:3D models rendered using an algorithm (Kim, 2008) 
The relevance of the NPR in graphic arts technology is supported also by the analytical approaches that attempt to implement objective evaluations of its use and quality of final visualizations in CG workflows. Author Isenberg (Isenberg, 2013) and his research group presented two types of evaluation methodologies (quantitative and qualitative) and the observation that NPR techniques are valuable to use, when they support a specific purpose. Furthermore, they presented an analytical study to examine the human perception on NRP visualisations and the differentiation in comparison with hand-drawn illustrations (Isenberg et al., 2006).

Tracing is an unappealing and time consuming process to artists. By using 3D graphics to aid with perspective a new problem has been created- the need to trace the 3D renders. In regards to this, the aim of the research was to develop the method to achieve a seamless integration of hand drawn 2D graphics and 3D (non-photorealistic) renders as they were rendered, creating the illusion of a completely hand drawn image and stylistic uniformity. The specific goals that would lead to this were: 1. removing the need to trace 3D models; 2 . finding the most appropriate technique for stylistic rendering of line art; 3 . determining a procedure for rendering line art drawings through computer models, so they would be stylistically uniform to hand drawn elements; 4 . ensuring a procedure that allows artists the freedom to create without compromising their style in order to accommodate the needs of computer software and 5 . visually seamless integration of drawn and rendered line art within the same drawing. We hypothesised that a new implemented method of creating line art elements using a render of a computer model is going to: 1 . from the point of the artist enable an easier creation of elements in perspective and enable a more correct creation of element in perspective; and 2. from the point of view of the observer enable a consistent creation of elements in perspective and convince observers that the entire image is a hand drawn image.

\section{Experimental}

\section{General plan of the research}

Once we had established the most appropriate software, Sketchup (Sketchup, 2015) (in our preliminary researches we discovered, that when we create a Sketchup style based on our line art and apply it to a model it looked like hand-drawn and it blended seamlessly with actual hand drawn lines), we planned out an analytical method by which we would test the effectiveness of its practical application. The research took place in two stages (Figure 3). First, we determined if our method is practical in simple applications with basic motifs. We createed simple motifs twice, once in $2 \mathrm{D}$ and once in 3D. Then we compared their likeness numerically with computer measurements and with the observation tests via a survey. The successfulness of the first phase was the condition to continue with the experimental and thus applying the process in the creation of an actual work of art (image with more complex motifs). After which we would evaluate our success via the results given to us by observers through a survey. In this final stage, the input of the observers was crucial and the observation test via a survey was performed for the second time. Finally, the critical analysis of the adequateness of the method, therefore the implemented procedure to fool the human eye into believing that the $3 \mathrm{D}$ renders were in fact drawn was carried out.

\section{MAKING BASIC MOTIFS}

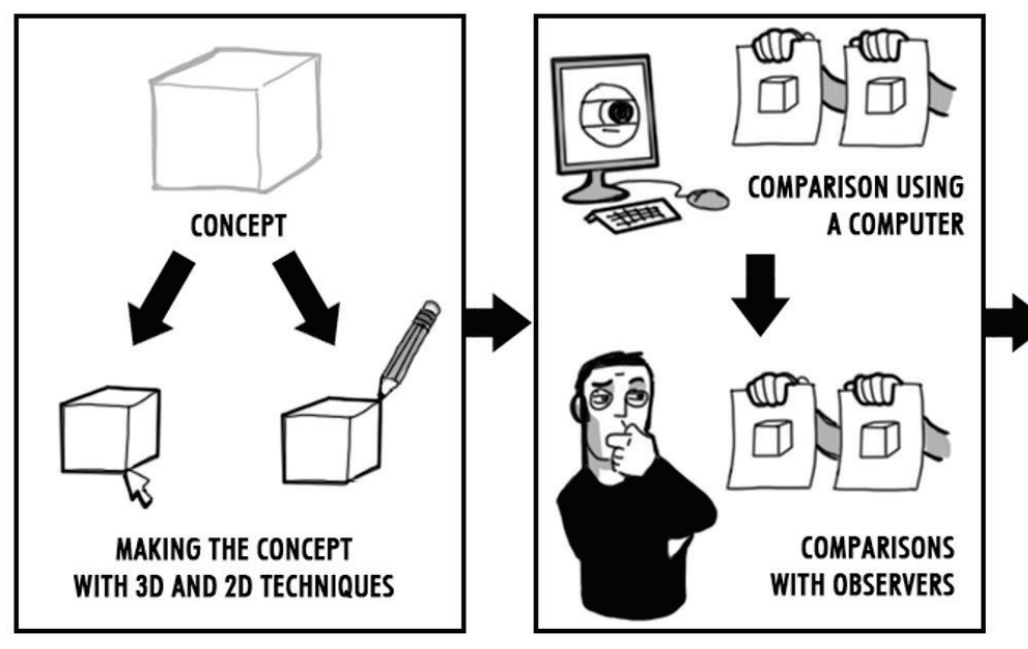

MAKING COMPLEX MOTIFS

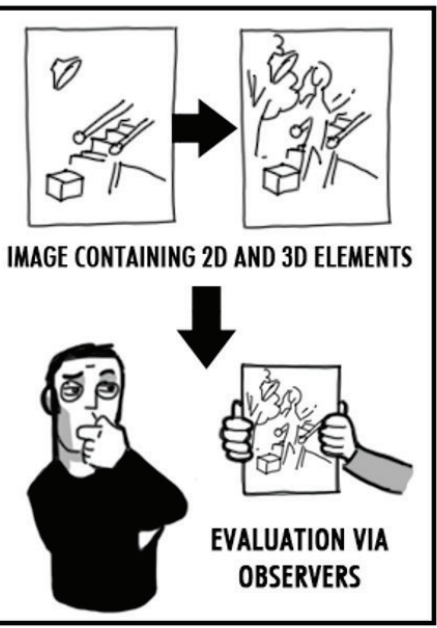

» Figure 3: General plan of the research 


\section{Determining the line art style}

The first stage of the experimental phase consisted of deciding on a line art style, which we would then attempt to imitate. Drawing and any kind of image editing was conducted in Photoshop through the Wacom Intuos 4 graphic tablet, whilst the 3D models were made in Sketchup Make. The Sketchup styles were created using Sketchup Style Builder.

Upon deciding on an art style, we exported a template from the Sketchup Style Builder. Style templates contain rectangles of differing lengths (in pixels of powers of two) in which line samples are inserted. These samples are used upon applying the Sketchup style to a 3D model. The sample length that optimally corresponds to the model edge length upon rendering, was automatically chosen. Both, the variety of length groups and the number of samples each group contains, could be adjusted. For the purposes of our selected art style, we choose six different lengths, each with five samples for variety. The finished Sketchup style, named 'Mark_1', contained 30 samples of unique hand drawn lines (Figure 4). There are also several additional options upon exporting from the Style Builder, which determine the behavior of the style, such as level of detail and depth cueing. Parameters presenting the behaviour of the style were adjusted accordingly to optimally fit the art style.

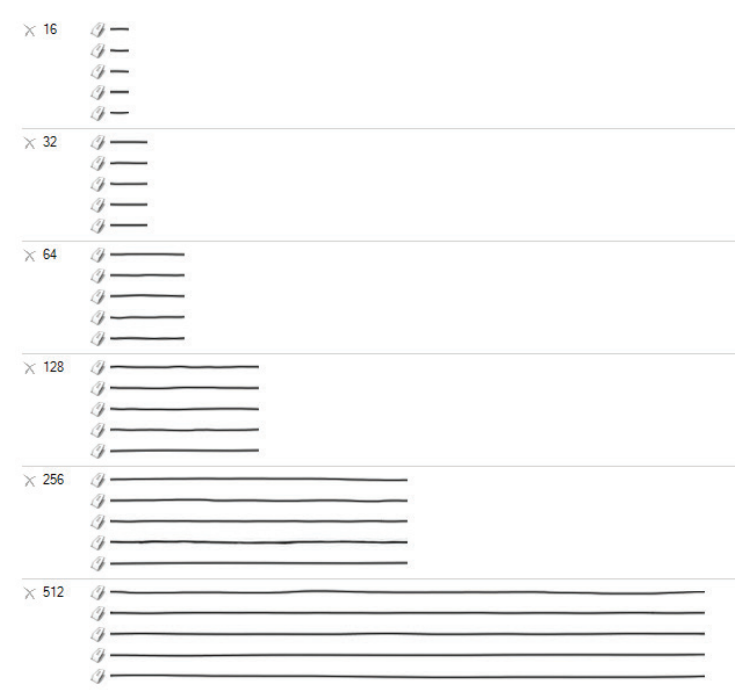

» Figure 4: Some of the lines contained within the style Mark_1

\section{Testing through basic geometrical motifs}

Before making a more complex image we had to test if we can emulate a drawing style on simple motifs with basic geometrical shapes and elements. The selected motifs were: lines, a triangle, a rectangle, a hexagon, an angular body, and a curved body (Figure 5). These were selected because they are archetypes of typical shapes and elements that appear in drawings: lines (free, crossing, and connecting), acute angles, obtuse angles, right angles, and curves. The motifs were then modeled in Sketchup and rendered using our Sketchup style Mark_1. They were also drawn in the same size in Photoshop using a graphic tablet.
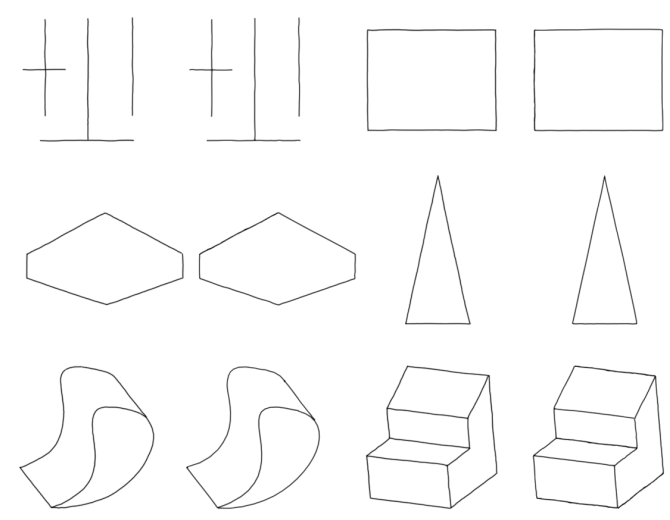

» Figure 5: 3D (left) and 2D (right) versions of simple motifs

To numerically quantify the similarity between the 3D renders and the $2 \mathrm{D}$ drawings we analyzed the images using an algorithm. The algorithm is based on the following foundations: if two shapes are identical, they would perfectly cover each other upon overlapping, and upon subtracting the value of one image from their composite the difference would be a blank image.

So if we define our rendered image as ' $u$ ', our drawn image as ' $r$ ', and our composite as ' $k$ ':

$(k-u=k-r) \leftrightarrow(u=r) \because(u+r=k)$

Alternatively, if we define ' $k-u$ ' as Ru and ' $k-r$ ' as Rr:

$(R u=R r) \leftrightarrow(u=r) \because(u+r=k)$

This also means that the more the two images would differ, the greater the surface area of their composite and the greater the values of Ru and Rr. The absolute difference between ' $R u^{\prime}$ ' and ' $R r^{\prime}$ ' is in effect the absolute difference between ' $u$ ' and ' $r$ '. If the absolute difference is normalized, we can then express the visual difference between two images in percentages. The algorithm is illustrated in scheme in Figure 6. It was written in Matlab, for the entire code see source (Arandjus, 2015).

As presented in Table 2 (Results), the differences between the $3 \mathrm{D}$ renders and their $2 \mathrm{D}$ counterparts turned out to be extremely small. Given that they are the same size and shape this was expected, but was now numerically quantified and gave us a mathematical justification to continue the experiment with human observers.

The experiment continued with a survey where we presented individuals with all six pairs of the motifs. 
The survey was done in person and through an online polling website. It was issued to students of Department of Textiles, Graphic Arts and Design at the Faculty of Natural Sciences and Engineering (University of Ljubljana), 3D modelers, it was posted on the forums of the 3D art website Sketchfab, sent to various artists, etc. The observation tests took place in interviewees home and office environment. All of them were done on personal computers through the website ' www.1ka. $\mathrm{si}^{\prime}$. They were unmonitored and undisturbed as to not sway their opinions even accidentally. We collected 110 completed surveys from laymen to people with various degrees of expertise in art and 3D graphics, thus ensuring a diverse range of participants.

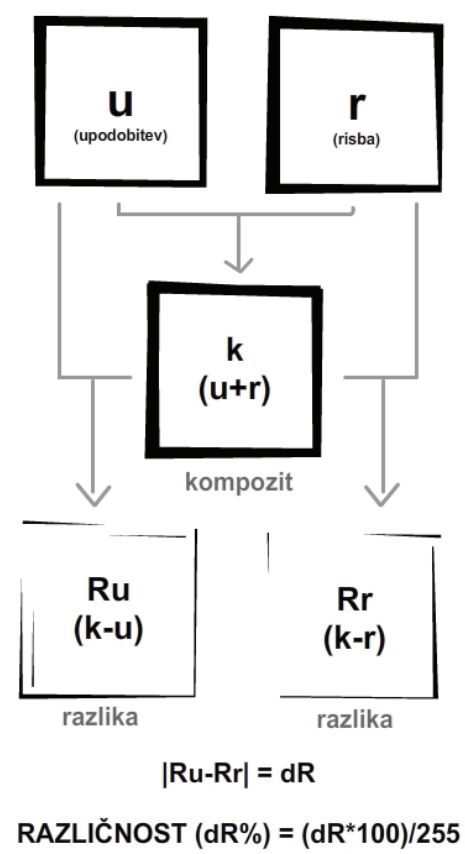

» Figure 6: Scheme illustrating how the algorithm works

In the observation tests with basic shapes, the observers were informed that one of the two motifs in the pair is a $3 \mathrm{D}$ render and they were instructed to simply choose which one they thought it was. The correct answer would give a value of 1 and the incorrect one a value of 0 . This means that, had the 3D render been completely obvious, the average result would be 1 or very close. Had the 3D render been similar to the drawing and observers could not tell them apart, they would have to guess. Meaning they would have a $50 \%$ chance of being correct and the results of the survey would be around 0.5. The results of the survey were statistically processed. An average was determined as well as the standard deviation (presented in Table 2). With the aim to confirm the method, we proceeded to make an actual complex illustration.

\section{Testing with a more complex illustration}

A complex illustration included several different types of shapes: from angular to curved, from organic to geometrical. These elements, both 2D and 3D, had to be in all corners of the image both in the background and in the foreground. The image also had to include a shadow, as the render of shadows is one of the advantages of using 3D graphics. The 3D shadows were also combined with 2D shadows to appear seamless. The image designed was that of a woman walking to her luggage, when the wind blows her hat away. The image itself was conceptualized to be in a three point perspective, a more advanced perspective type (Artyfactory, 2015) which would justify the need to use 3D graphics as an aid. The list of elements used in the illustration with their descriptions is presented in Table 1.

Upon deciding on these elements, we proceeded with creating the illustration (Figures 7-10). We began with a sketch. Based on the sketch we made and lit the 3D model. We then rendered the model and shadows into separate images. After that we imported the renders into Photoshop where we drew the remaining 2D elements (lines and shadows) on separate layers.

The application of Sketchup styles was unfortunately not perfect (Figure 9). There were small errors. In certain places the lines went too far and some short lines are not rendered at all. This was of course an undesired, but it easily fixable, effect. It could be argued that these mistakes contribute to a sort of visual humanization.

\section{Table 1}

List of elements and their descriptions in the complex illustration

\begin{tabular}{|c|c|c|c|}
\hline \multicolumn{2}{|l|}{ 3D elements } & \multicolumn{2}{|l|}{ Drawn elements } \\
\hline Element & Description & Element & Description \\
\hline Steps & angular geometrical element & Woman & irregular organic element \\
\hline Building with windows & angular geometrical element & Plant & irregular organic element \\
\hline Pot & round geometrical element & Gift box bow & curvy geometrical element \\
\hline Violin case & curvy geometrical element & Travel bag & round organic element \\
\hline Gift box and briefcase & angular geometrical element & Tree & irregular organic element \\
\hline Fence in front of the building & repeating vertical pattern & Leaves & curvy geometrical element \\
\hline Sidewalk tiles & grid pattern & Curtains in the window & irregular organic element \\
\hline Hat & round organic element & Decals and handles on the luggage & round geometrical elements \\
\hline Most shadows & irregular organic element & Various wear and tear & smaller details \\
\hline
\end{tabular}




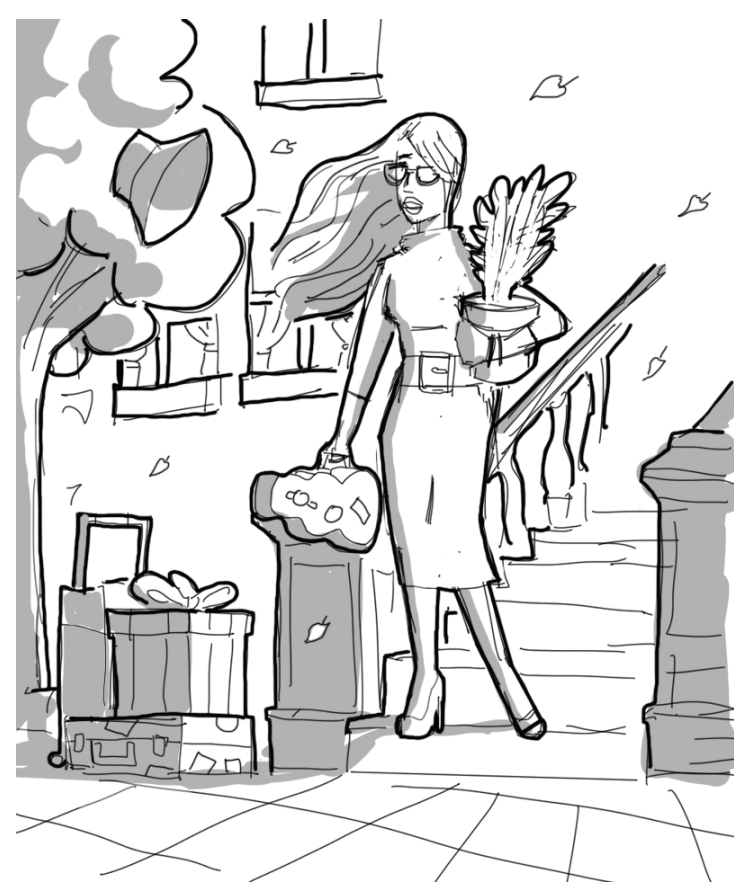

» Figure 7: Initial sketch

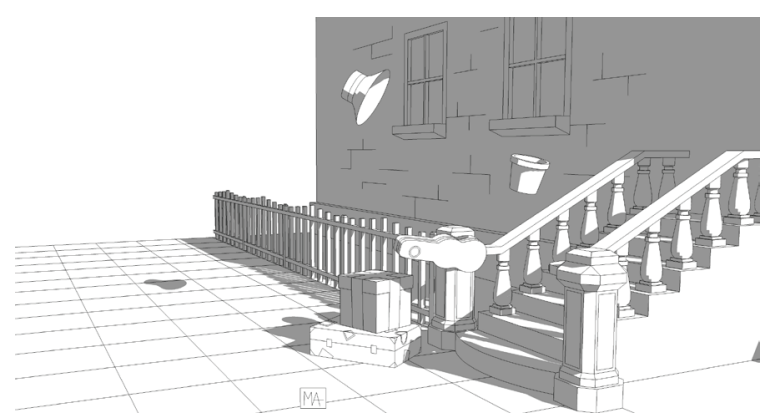

» Figure 9: The finished 3D scene, complete with shadows, before applying the Sketchup style

Humanization is an effect that originates from digital music and includes a deliberate addition of small errors in the performance of a programmed instrument to create a more natural output result (Hennig et al., 2011). In our illustrations the errors in rendering can help with making the $3 \mathrm{D}$ render appear more hand-drawn, since artists make similar errors when drawing.

After finalizing the complex illustration, the observation test was performed again (the performance of survey testing is presented above). The key points of success upon which we measured the integration of $3 D$ and $2 D$ were:

- Firstly, how similar were the lines. Did it seem as if it was drawn by the same person? This was tested with question 1: "Did you think the image is stylistically uniform (particularly regarding the similarity of the lines)? « The available answers were: 1 . completely; 2 . very; 3. largely; 4 . slightly and 5 . not in the least.

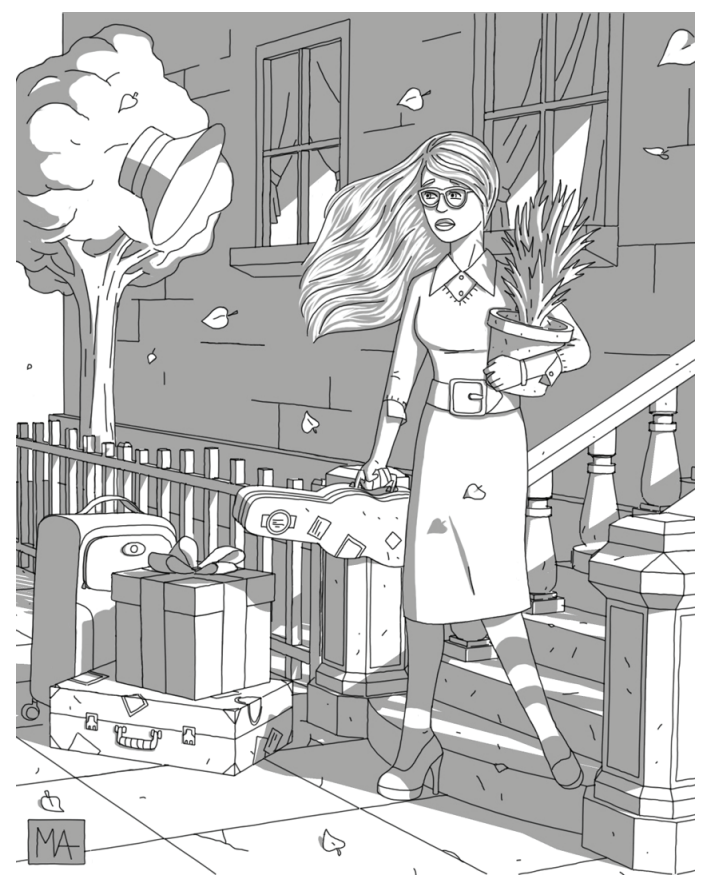

» Figure 8: Finished illustration

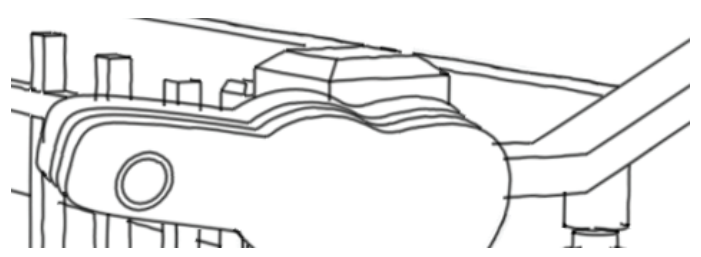

» Figure 10: Imperfections in the application of Sketchup styles

- Secondly, does the image appear drawn, given the fact that this is one of the main effects we were trying to achieve? This was tested with question 2: »In the beginning it was stated several times that the image is a drawing. Upon viewing the image, did you think it was a drawing? « The available answers were: 1 . Yes, the image seemed completely drawn; 2 . I had doubts, whether it was completely drawn and 3. No, the image did not seem completely drawn.

- Finally, we informed the participants: »In truth, the image is only partially drawn; many of the elements of the image are renders of 3D models." and instructed them: "If you suspected this while viewing the image, write which parts seemed like they were 3D graphics, otherwise leave blank«, upon which they were presented with an optional text field. It was important to let the participants describe any part of the image they wished without hinting to which parts could be 3D graphics. 
The results of questions 1 and 2 are represented both in actual numbers as well as percentage-wise. The answers to the third question (where they were asked to identify which parts of the image seemed as 3D graphics), have been transcribed into with the results of the first two questions in the following columns for comparison. The specifics of their answers were later compared, to see if there were any similarities between them or other details that might be of interest.

\section{Results}

\section{Results of measuring simple motifs}

Table 2 shows the results of measurements done through the difference algorithm we wrote to measure simple motifs, while Table 3 shows the results of the survey we presented, to determine if observers could distinguish $2 \mathrm{D}$ and $3 \mathrm{D}$ in regards to simple motifs. The results were around 0.5 and participants often remarked they were simply choosing at random and could not tell the difference. Consequently, the assumption that Sketchup styles can fool the human eye was confirmed.

Table 2

Results of comparisons via algorithm

\begin{tabular}{l|c|c|c}
\hline Motif & $\mathbf{R u}$ & $\mathbf{R r}$ & Difference (\%) \\
\hline Lines & 2.04 & 2.19 & $0.0597 \%$ \\
\hline Rectangle & 2.25 & 2.37 & $0.0459 \%$ \\
\hline Hexagon & 1.76 & 1.82 & $0.0222 \%$ \\
\hline Triangle & 1.86 & 1.98 & $0.0475 \%$ \\
\hline Angular body & 3.89 & 3.94 & $0.0196 \%$ \\
\hline Curvy body & 2.94 & 3.21 & $0.1046 \%$ \\
\hline
\end{tabular}

Table 3

Results of the observation test for simple motifs

\begin{tabular}{l|c|c|c|c}
\hline Motif & Average & $\begin{array}{c}\text { Standard } \\
\text { deviation }\end{array}$ & Minimum & Maximum \\
\hline Lines & 0.450 & 0.501 & & \\
\cline { 1 - 3 } Rectangle & 0.563 & 0.499 & & \\
\cline { 1 - 3 } Hexagon & 0.438 & 0.499 & \multirow{2}{*}{0} & \multirow{2}{*}{1} \\
\cline { 1 - 3 } Triangle & 0.650 & 0.480 & & \\
\cline { 1 - 2 } Angular body & 0.450 & 0.501 & & \\
\hline Curvy body & 0.400 & 0.493 & & \\
\hline
\end{tabular}

\section{Table 4}

Results of the first question: »Did you think the image is stylistically uniform (particularly regarding the similarity of the lines)? ?

\begin{tabular}{l|c|c}
\hline Answer & Number of answers & \% \\
\hline completely & 39 & 35 \\
\hline very & 52 & 47 \\
\hline largely & 17 & 15 \\
\hline slightly & 2 & 2 \\
\hline not in the least & 0 & 0 \\
\hline
\end{tabular}

\section{Results of measuring the complex motif}

Table 4 and 5 contain the numbers of chosen answers of the first two questions in the survey, regarding people's perception of the final created digital illustration. Table 6 contains the comments some observers had, regarding the third question as well as the answers these observers chose in the first two questions.

\section{Table 5}

Results of the second question: »In the beginning it was stated several times that the image is a drawing. Upon viewing the image, did you think it was a drawing?"

\begin{tabular}{l|c|c}
\hline Answer & $\begin{array}{c}\text { Number of } \\
\text { answers }\end{array}$ & \% \\
\hline $\begin{array}{l}\text { Yes, the image seemed } \\
\text { completely drawn. }\end{array}$ & 64 & 58 \\
\hline $\begin{array}{l}\text { I had doubts, whether it } \\
\text { was completely drawn. }\end{array}$ & 24 & 22 \\
\hline $\begin{array}{l}\text { No, the image did not } \\
\text { seem completely drawn. }\end{array}$ & 22 & 20 \\
\hline
\end{tabular}

\section{Table 6}

Comments at the second question, as well as the answers given in the first two

\begin{tabular}{l|c|c}
\hline Comment & $\begin{array}{c}\text { Answer for } \\
\text { question 1 }\end{array}$ & $\begin{array}{c}\text { Answer for } \\
\text { question 2 }\end{array}$ \\
\hline $\begin{array}{l}\text { »The railing on the stairs } \\
\text { seemed too correct- as } \\
\text { if a reference photo was } \\
\text { used when it was drawn } \\
\text { or perhaps it was traced « }\end{array}$ & very & 3 \\
$\begin{array}{l}\text { »ust seemed like } \\
\text { certain parts were } \\
\text { a bit different." }\end{array}$ & very & \\
\hline
\end{tabular}

\section{Table 7}

Results of the third question, as well as the answers given in the first two

\begin{tabular}{|c|c|c|}
\hline Comment & $\begin{array}{l}\text { Answer for } \\
\text { question } 1\end{array}$ & $\begin{array}{l}\text { Answer for } \\
\text { question } 2 \\
\end{array}$ \\
\hline "Gift box« & very & 2 \\
\hline $\begin{array}{l}\text { »Hat by the tree, } \\
\text { suitcases, present." }\end{array}$ & largely & 2 \\
\hline »Fence, suitcase» & very & 2 \\
\hline $\begin{array}{l}\text { »Mostly I noticed the gift } \\
\text { box that had too perfect } \\
\text { lines and I doubt anybody } \\
\text { has such a steady hand « }\end{array}$ & very & 2 \\
\hline »elements on the cases» & largely & 3 \\
\hline $\begin{array}{l}\text { »suitcase, aparte- } \\
\text { ment, the model« }\end{array}$ & very & 2 \\
\hline $\begin{array}{l}\text { »the hat and the } \\
\text { boxes/case« }\end{array}$ & very & 3 \\
\hline
\end{tabular}




\section{Disscusion}

From the creator's point of view, an implemented method of creating line art elements using a render of computer models does enable an easier creation of elements in perspective. It also enables a more correct creation of elements in perspective. The presented method enabled a consistent creation of elements in perspective and the results of its implementation convinced the majority of observers that the entire image was hand drawn. Our hypotheses were confirmed.

The implementation of an algorithm in the analysis gave satisfactory results. The algorithmic measurements gave an extremely small percentage of difference between the 2D and 3D variants of simple motifs (Table 2). The biggest difference was a mere tenth of a percent; the motif was the curved body. This indicated that, when modeling curvy elements, the creator must pay closer attention to ensure the desired effect of emulating $2 \mathrm{D}$ line art.

In the first survey, where we asked participants to choose between a 3D render and a 2D drawing, there was no significant trend one way or the other. Consistently it was an average of around 0.5 , something one would expect to see if the answers were chosen at random. This is further confirmed by the fact that on many occasions after completing the survey the participants told us that they didn't know which was which and were simply guessing. Both the algorithm and the survey confirmed that one can use Sketchup to emulate drawn shapes to a high degree of similarity.

In the first part of the research, some defined goals were achieved. The need to trace 3D models was removed from the procedure, the optimal technique for stylistic rendering of line art was determined (with the digitalisation of personal line-art style and the implementation in defined software) and, finally, a defined procedure for rendering line art drawings through computer models was confirmed to result in style uniformity of simple motifs in comparison to hand drawn lines.

In the next phase we measured the observers perception of the more complex image with the lady and the plant. Regardless the complexity of the illustration, the results of the first question confirmed that most observers perceived the combination of line-arts as stylistically uniform. Here, most observers (91 people, 81\% of answers) were of the opinion that the image is at least very stylistically uniform. These results were quite satisfactory and confirmed that using Sketchup styles is effective at maintaining stylistic uniformity when combining 2D drawings and $3 \mathrm{D}$ renders. Looking into the results of the second question we can see, that most people (64\%) perceived the image as completely drawn. Almost a quarter (24\%) had doubts whether or not it was completely drawn, but was not sure. Around a fifth (22\%) did not see the image as completely drawn. These results allude to an explanation that the emulation of drawing by hand is not perfect and that a portion of observers will never fully perceive the image as drawn. However, out of the ones that claimed they did not feel the image was completely drawn, only two answered that they noticed the presence of 3D graphics (Table 6). Overall, the results of the second question tell us that, while not perfect, our method was mostly successful in emulating drawn line art.

In regards to question 3, the results show very few noticed the presence of 3D graphics, and amongst those who did, all noticed but a few did choose the right elements (some elements were incorrectly identified). The final image included eight elements which were renders of 3D models. Out of the 110 participants only seven people (8\%) noted the presence of 3D graphics. Out of these seven, four incorrectly identified a 2D (or partially 2D) object as 3D; they could not tell the difference between which was which. The observers did not identified the steps, the signature, the pot, the windows or the sidewalk as a 3D element, meaning 5 out of 8 3D elements in the image went unnoticed by everyone. Likewise, nobody noticed the shadows, which were largely exported from 3D computer graphic software. Given these results, we can conclude that the use of Sketchup styles to render 3D graphics non-photorealistically is virtually seamless in regards to the $2 \mathrm{D}$ elements within the same image. In this second phase, the remaining goals were achieved. The proposed method ensured the creation of line-art illustrations without compromising the author's style and the seamless integration of drawn and rendered line art within the same drawing.

\section{Conclusions}

The results of the research revealed that people rarely notice the presence of 3D graphics and even if they do, they are not able to claim with certainty weather the element is $2 \mathrm{D}$ or $3 \mathrm{D}$. In the presented research we have developed, successfully applied, and confirmed a new method of integrating stylized 2D and 3D elements seamlessly in the same digital illustration and, consequently, removed any need for tracing. The process does not limit the artist to make compromises with his style on his behalf, as the software adapts to the artist per our setting and not the other way around. The process enabled an easier creation of an image that contains complex perspective, since making the 3D models was faster, easier and more correct than it would have been to draw them. Our aims have hence been reached, completing our goal of removing the need to trace 3D renders. The results of the research would allow many artists to save time and effort in creating images which they would otherwise be unable to, or would shy away for being too challenging. The presented method for rendering line art drawings through computer models 
ensures that the computer drawings are stylistically uniform to hand drawn elements and the freedom to create without compromising the artist's style in order to accommodate the needs of computer software. Moreover, the procedure enables visually seamless integration of drawn and rendered line art within the same drawing.

\section{References}

Aladdin. (1992) [Animated film] Directed by: Ron Clements \& John Musker. USA, Walt Disney Feature Animation.

Arandjus, M. (2015) Rendering Three-Dimensional Computer Graphics as an Aid to Stylized Line Drawings in Perspective. MSc thesis. University of Ljubljana.

Artyfactory. (2015) Perspective Drawing- Three Point Perspective. Available from: http://www.artyfactory.com/ perspective_drawing/perspective_5.html [Accessed 18th June 2015].

Bousseau, A., Neyret, F., Thollot, J. \& Salestin, D. (2007) Video Water colorization Using Bidirectional Texture Advection. ACM Transactions on Graphics. 26 (3), 104104:7. Available from: doi: 10.1145/1276377.1276507 [Accessed 10th May 2016].

Family Guy. (2010) [Animated series] Los Angeles, Fox Broadcasting Company.

Hennig, H., Fleischmann, R., Fredebohm, A., Hagmayer, Y., Nagler, J., Witt, A., Theis J. F. \& Geisel T. (2011) The nature and perception of Fluctuations in Human Musical Rhythms. PLOS ONE. 6 (10), 1-7. Available from: doi: 10.1371/journal.pone.0026457 [Accessed 18th July 2015].

Isenberg, T. (2013) Evaluating and Validating Non-Photorealistic and Illustrative Rendering. In: Rosin, P. \& Collomosse, J. (eds.) Image and Video based Artistic Stylisation. London, Springer. pp. 311-331.

Isenberg, T., Neuman, P., Carpendale, S., Costa Sousa, M. \& Jorge, J.A. (2006) Non-Photorealistic Rendering in Contex: An Observational Study. In: DeCarlo, D. \& Markosian, L. (eds.) NPAR 2006: Proceedings of the Fourth International Symposium on Non-Photorealistic Animation and Rendering, NPAR 2006, 05 - 07 June, Annecy, France. New York, ACM. pp. 115-126.

Kalinins, D., Davidson, P., Marksian, L. \& Finkelstein, A. (2009) Coherent Stylized Silhouettes. Available from: http://gfx.cs.princeton.edu/pubs/Kalnins_2003_css/ kalnins2003css.pdf [Accessed 25th June 2016].
Kang, H., Lee, S. \& Chui, C. (2009) Flow-Based Image Abstraction. IEEE Transactions on Visualisation and Computer Graphics. 15 (1), 62-76. Available from: doi: 10.1109/TVCG.2008.81 [Accessed 22nd June 2016].

Kim, Y., Yu, J., Yu, X. \& Lee, S. (2008) Line-art Illustration of Dynamic and Specular Surfaces. ACM Transactions on Graphics. 27 (5), 156-165. Available from: doi: 10.1145/1409060.1409109 [Accessed 22nd June 2016].

Kyprianidis, J.E., Kang, H. \& Döllner J. (2009) Image and Video Abstraction by Anisotropic Kuwahara Filtering. Computer Graphics Forum. 28 (7), 1955-1963. Available from: doi: 10.1111/j.1467-8659.2009.01574.x [Accessed 22nd May 2016].

Ni no Kuni. (2010) [Computer game] Fukuoka, Level-5.

Paperman. (2012) [Animated short] Directed by: John Kahrs. USA, Walt Disney Animation Studios.

Robertson, B. (1997) Different Strokes. Computer Graphics World. 20 (12), 26

Sketchup. (2015) Features: Presentation. Available from: http://www.sketchup.com/products/sketchup-pro/ features/presentation [Accessed 4th July 2015].

Tarzan. (1999) [Animated film] Directed by: Chris Buck \& Kevin Lima. USA, Walt Disney Studios.

Tarzan. (Disney Collector's Edition) (2000) [DVD-ROM] Burbank, Walt Disney Studios.

The Iron Giant. (1999) [Animated film] Directed by: Brad Bird. USA, Warner Bros. Animation.

The Making of 'Aladdin': a Whole New World. (1992) [Documentary film] Directed by: Stephen Kroopnick. USA, Walt Disney Home Video.

Way, D.L., Yang, M.K., Shih, Z.C \& Lee, R.R. (2014) A Colored Pencil Non-Photorealistic rendering for 2D Images. International Journal of Innovative Computing, Information and Control. 10 (1), 233-241. Available from: http://www.ijicic.org/ijicic-12-10039. pdf [Accessed 10th July 2016].

Wilson, M. (2013) Why This Oscar-Winning Disney Short Looks Like Nothing Made Before. Available from: http://www.fastcodesign.com/1671941/why-this-oscar-nominated-disney-short-looks-like-nothing-madebefore [Accessed 9th December 2014].

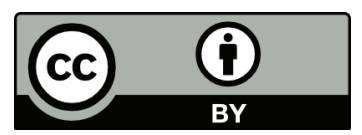

(C) 2016 Authors. Published by the University of Novi Sad, Faculty of Technical Sciences, Department of Graphic Engineering and Design. This article is an open access article distributed under the terms and conditions of the Creative Commons Attribution license 3.0 Serbia (http://creativecommons.org/licenses/by/3.0/rs/). 